\title{
VARIATIONS ON A-BROWDER-TYPE THEOREMS
}

\author{
M. BERKANI*, M. KACHAD, H. ZARIOUH AND H. ZGUITTI
}

\begin{abstract}
We introduce and we study the new spectral properties $(S B w),(S B a w),(S B a b)$ and $(S B b)$. Among other results, we show that if $T$ is a bounded linear operator acting on a Banach space $X$, then $T$ possesses property $(S B b)$ if and only if $T$ possesses property $(b)$ and $\Pi^{0}(T)=\Pi_{a}(T)$.
\end{abstract}

\section{INTRODUCTION}

This paper is a continuation of our recent investigations in the subject of Weyl type theorems. As in [12], we investigate other new variants of a-Browder's and a-Weyl's theorem, and we define the properties $(S B w)$, $(S B b),(S B a b)$ and $(S B a w)$ (see Definitions 2.1 and 2.10) for bounded linear operators, in connection with Weyl-type theorems. The essential results obtained are summarized in the diagram presented in the last part of this paper (see Table 1). For further definitions and symbols we refer the reader to [12], and we also refer to $[4,5,6,9,11,13]$ for more details. The inclusion of the meaning of various known theorems and properties (see Table 2) is motivated by having a global overview of the subject. In addition, we have the following usual notations that will be needed later:

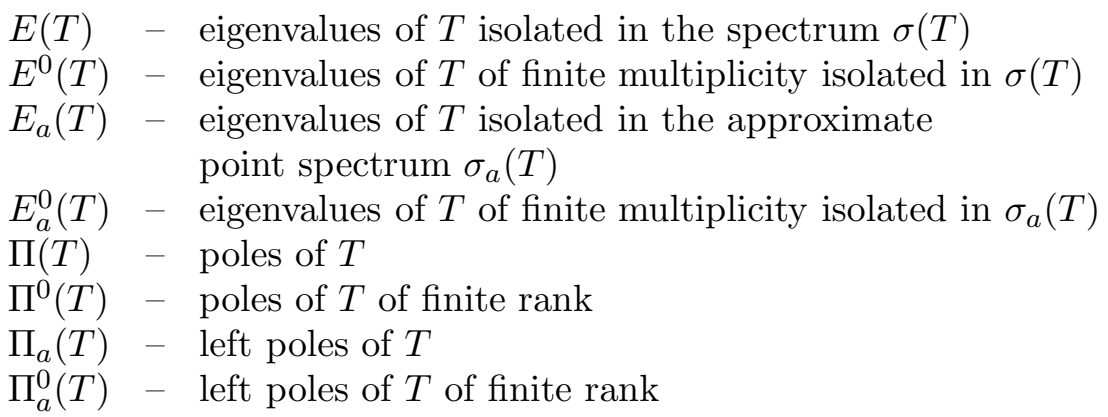

2010 Mathematics Subject Classification. Primary 47A53, 47A10, 47A11.

Key words and phrases. Essential semi-B-Fredholm spectrum, a-Weyl's theorem, aBrowder's theorem, property $(S B w)$.

* Supported by Protars D11/16 and PGR- UMP. 


$$
\begin{array}{ll}
\sigma_{B W}(T) & - \text { B-Weyl spectrum of } T \\
\sigma_{W}(T) & - \text { Weyl spectrum of } T \\
\sigma_{S B F_{+}^{-}}(T) & - \text { essential semi-B-Fredholm spectrum of } T \\
\sigma_{S F_{+}^{-}}(T) & - \text { Weyl essential approximate point spectrum of } T
\end{array}
$$

\section{MAin RESUlts}

We denote by $L(X)$ the Banach algebra of all operators acting on a Banach space $X$. For $T \in L(X)$, let $\Delta_{a}(T)=\sigma_{a}(T) \backslash \sigma_{S F_{+}^{-}}(T), \Delta_{a}^{g}(T)=$ $\sigma_{a}(T) \backslash \sigma_{S B F_{+}^{-}}(T)$ and let $\Delta^{g}(T)=\sigma(T) \backslash \sigma_{B W}(T)$.

An operator $T \in L(X)$ is said to have the single valued extension property at $\lambda_{0} \in \mathbb{C}$ (abbreviated SVEP at $\lambda_{0}$ ), if for every open neighborhood $\mathcal{U}$ of $\lambda_{0}$, the only analytic function $f: \mathcal{U} \longrightarrow X$ which satisfies the equation $(T-\lambda I) f(\lambda)=0$ for all $\lambda \in \mathcal{U}$ is the function $f \equiv 0$. An operator $T \in L(X)$ is said to have the SVEP if $T$ has this property at every $\lambda \in \mathbb{C}$. (See [16] for more details about this concept).

Definition 2.1. A bounded linear operator $T \in L(X)$ is said to possess property $(S B w)$ if $\Delta_{a}^{g}(T)=E^{0}(T)$ and is said to possess property $(S B b)$ if $\Delta_{a}^{g}(T)=\Pi^{0}(T)$.

Recall that $T \in L(X)$ satisfies property $(w)$ if $\sigma_{a}(T) \backslash \sigma_{S F_{+}^{-}}(T)=E^{0}(T)$.

Theorem 2.2. Let $T \in L(X)$. Then $T$ possesses property $(S B w)$ if and only if $T$ possesses property $(w)$ and $\sigma_{S B F_{+}^{-}}(T)=\sigma_{S F_{+}^{-}}(T)$.

Proof. Suppose that $T$ possesses property $(S B w)$, that is $\Delta_{a}^{g}(T)=E^{0}(T)$. Since $\Delta_{a}(T) \subset \Delta_{a}^{g}(T)$ then $\Delta_{a}(T) \subset E^{0}(T)$. Now if $\lambda \in E^{0}(T)$, then $T-\lambda I$ is an upper semi-B-Fredholm operator. As $\lambda$ is an eigenvalues of $T$ of finite multiplicity, then from [11, Lemma 2.2], $T-\lambda I$ is an upper semiFredholm operator. Thus $\Delta_{a}(T) \supset E^{0}(T)$. Hence $T$ possesses property $(w)$. We then have $\sigma_{S B F_{+}^{-}}(T)=\sigma_{a}(T) \backslash E^{0}(T)$ and $\sigma_{S F_{+}^{-}}(T)=\sigma_{a}(T) \backslash E^{0}(T)$. So $\sigma_{S B F_{+}^{-}}(T)=\sigma_{S F_{+}^{-}}(T)$.

Conversely, the condition $\sigma_{S B F_{+}^{-}}(T)=\sigma_{S F_{+}^{-}}(T)$ entails that $\Delta_{a}^{g}(T)=\Delta_{a}(T)$. The property $(w)$ for $T$ implies that $\Delta_{a}^{g}(T)=E^{0}(T)$ and $T$ possesses property $(S B w)$.

Generally, property $(w)$ does not imply property $(S B w)$. Indeed, let $U \in$ $L\left(\ell^{2}(\mathbb{N})\right)$ be defined by $U\left(x_{1}, x_{2}, x_{3}, \ldots\right)=\left(0, x_{2}, x_{3}, \ldots\right)$. Then $\sigma_{a}(U)=$ $\{0,1\}, \sigma_{S F_{+}^{-}}(U)=\{1\}$ and $E^{0}(U)=\{0\}$. Thus $\Delta_{a}(U)=E^{0}(U)$, i.e. $U$ possesses property $(w)$. Moreover, $\sigma_{S B F_{+}^{-}}(U)=\emptyset$. This implies that $\Delta_{a}^{g}(U) \neq E^{0}(U)$ and $U$ does not possess property $(S B w)$. 
Remark 2.3. 1) The property $(S B w)$ is not intermediate between property $(w)$ and property $(g w)$. Indeed, let $T=0 \oplus S$ be defined on the Banach space $\ell^{2}(\mathbb{N}) \oplus \ell^{2}(\mathbb{N})$, where $S$ is defined on $\ell^{2}(\mathbb{N})$ by $S\left(x_{1}, x_{2}, x_{3}, \ldots\right)=$ $\left(0, \frac{1}{2} x_{1}, \frac{1}{3} x_{2}, \ldots\right)$. Then $\sigma_{a}(T)=\sigma_{S B F_{+}^{-}}(T)=\{0\}$ and $E(T)=\{0\}$. So $\Delta_{a}^{g}(T) \neq E(T)$, i.e. $T$ does not possesses property $(g w)$. On the other hand, since $E^{0}(T)=\emptyset$ we have $\Delta_{a}^{g}(T)=E^{0}(T)$, i.e. $T$ possess property $(S B w)$. Now if we consider the operator $U$ defined above, then $E(U)=\{0,1\}$. So $\Delta_{a}^{g}(U)=E(U)$, i.e. $U$ possesses property $(g w)$. But it does not possess property $(S B w)$.

2) Generally, the property $(S B w)$ is not transmitted from an operator $T$ to its dual $T^{*}$. To see this, consider the operator $S$ be defined as in part 1) of this remark, then $S$ possesses property $(S B w)$ because $\sigma_{a}(S)=$ $\sigma_{S B F_{+}^{-}}(S)=\{0\}$ and $E^{0}(S)=\emptyset$. But its adjoint which is defined by $S^{*}\left(x_{1}, x_{2}, x_{3}, \ldots\right)=\left(\frac{1}{2} x_{2}, \frac{1}{3} x_{3}, \frac{1}{4} x_{4}, \ldots\right)$ does not possess this property because $\sigma_{a}\left(S^{*}\right)=\sigma_{S B F_{+}^{-}}\left(S^{*}\right)=\{0\}$ and $E^{0}\left(S^{*}\right)=\{0\}$.

From Theorem 2.2, we deduce immediately that an operator possessing property $(S B w)$ possesses property $(S B b)$, but the converse does not hold in general as shown by the following example: let $T$ be the operator be defined on the Hilbert space $\ell^{2}(\mathbb{N})$ by $T\left(x_{1}, x_{2}, x_{3}, \ldots\right)=\left(\frac{1}{2} x_{2}, \frac{1}{3} x_{3}, \frac{1}{4} x_{4}, \ldots\right)$. Then $\sigma_{a}(T)=\sigma_{S B F_{+}^{-}}(T)=\{0\}, E^{0}(T)=\{0\}$ and $\Pi^{0}(T)=\emptyset$. This implies that $\Delta_{a}^{g}(T)=\Pi^{0}(T)$ and $\Delta_{a}^{g}(T) \neq E^{0}(T)$, so that $T$ possesses property $(S B b)$, but it does not possess property $(S B w)$. However, we have the following corollary in which we give a condition for the equivalence of these properties.

Corollary 2.4. Let $T \in L(X)$. Then $T$ possesses property $(S B w)$ if and only if $T$ possesses property $(S B b)$ and $E^{0}(T)=\Pi^{0}(T)$.

The next theorem gives characterizations of operators possessing property $(S B b)$. Recall that $T \in L(X)$ satisfies property $(b)$ (respectively property $(g b))$ if $\sigma_{a}(T) \backslash \sigma_{S F_{+}^{-}}(T)=\Pi^{0}(T)$ ( respectively: $\left.\sigma_{a}(T) \backslash \sigma_{S B F_{+}^{-}}(T)=\Pi(T)\right)$.

Theorem 2.5. Let $T \in L(X)$. Then the following statements are equivalent.

(i) $T$ possesses property $(S B b)$;

(ii) $T$ possesses property (b) and $\sigma_{S B F_{+}^{-}}(T)=\sigma_{S F_{+}^{-}}(T)$;

(iii) $T$ possesses property (b) and $\Pi^{0}(T)=\Pi_{a}(T)$;

(iv) $T$ possesses property $(g b)$ and $\Pi^{0}(T)=\Pi(T)$.

Proof.

(i) $\Longleftrightarrow$ (ii). Suppose that $T$ possesses property $(S B b)$, that is $\Delta_{a}^{g}(T)=$ $\Pi^{0}(T)$. Since $\Delta_{a}(T) \subset \Delta_{a}^{g}(T), \Delta_{a}(T) \subset \Pi^{0}(T)$. As $\Delta_{a}(T) \supset \Pi^{0}(T)$ is always true, then $\Delta_{a}(T)=\Pi^{0}(T)$ and $T$ possesses property $(b)$. We then have 
$\sigma_{S B F_{+}^{-}}(T)=\sigma_{a}(T) \backslash \Pi^{0}(T)$ and $\sigma_{S F_{+}^{-}}(T)=\sigma_{a}(T) \backslash \Pi^{0}(T)$. So $\sigma_{S B F_{+}^{-}}(T)=$ $\sigma_{S F_{+}^{-}}(T)$. Conversely, the condition $\sigma_{S B F_{+}^{-}}(T)=\sigma_{S F_{+}^{-}}(T)$ and property $(b)$ for $T$ imply that $\Delta_{a}^{g}(T)=\Pi^{0}(T)$ and $T$ possesses property $(S B b)$.

(i) $\Longleftrightarrow$ (iii). Assume that $T$ possesses property $(S B b)$, then $T$ possesses property $(b)$. From [11, Theorem 2.5], $T$ satisfies a-Browder's theorem, and since a-Browder's theorem is equivalent to generalized a-Browder's theorem then $\Delta_{a}^{g}(T)=\Pi_{a}(T)$. Hence $\Pi^{0}(T)=\Pi_{a}(T)$. Conversely, assume that $T$ possesses property $(b)$ and $\Pi^{0}(T)=\Pi_{a}(T)$. Then $\Delta_{a}^{g}(T)=\Pi_{a}(T)$ and $\Pi^{0}(T)=\Pi_{a}(T)$. Thus $\Delta_{a}^{g}(T)=\Pi^{0}(T)$ and $T$ possesses property $(S B b)$.

(i) $\Longleftrightarrow$ (iv). If $T$ possesses property $(S B b)$, that is $\Delta_{a}^{g}(T)=\Pi^{0}(T)$, then $\Delta_{a}^{g}(T) \subset \Pi(T)$. Since we have always that $\Delta_{a}^{g}(T) \supset \Pi(T)$, then $\Delta_{a}^{g}(T)=$ $\Pi(T)=\Pi^{0}(T)$. So $T$ possesses property $(g b)$. The reverse implication is trivial.

Remark 2.6. 1) From Theorem 2.5, we have if $T \in L(X)$ possesses property $(S B b)$ then it possesses property $(g b)$. However, the converse is not true in general: for example, let $T$ be defined on $\ell^{2}(\mathbb{N})$ by $T\left(x_{1}, x_{2}, x_{3}, \ldots\right)=$ $\left(0, \frac{1}{2} x_{1}, 0,0, \ldots\right)$. Then $\sigma_{a}(T)=\{0\}, \sigma_{S B F_{+}^{-}}(T)=\emptyset$ and $\Pi(T)=\Pi_{a}(T)=$ $\{0\}$. So $\Delta_{a}^{g}(T)=\Pi(T)$, i.e. $T$ possesses property $(g b)$. But it does not possess property $(S B b)$, since $\Pi^{0}(T)=\emptyset$. Here $\sigma_{S F_{+}^{-}}(T)=\{0\}$.

2) Property $(S B b)$ as well as property $(S B w)$, does not pass from an operator to its dual. For this, we consider on the Hilbert space $\ell^{2}(\mathbb{N})$ the operator $T$ defined by $T\left(x_{1}, x_{2}, x_{3}, \ldots\right)=\left(\epsilon x_{1}, 0, x_{2}, x_{3}, \ldots\right)$ for fixed $0<\epsilon<1$. Then $\sigma(T)=D(0,1)$ the closed unit disc in $\mathbb{C}$ and $\sigma_{a}\left(T^{*}\right)=C(0,1) \cup\{\epsilon\}$ where $C(0,1)$ is the unit circle of $\mathbb{C}$. This implies that $T^{*}$ has the SVEP, which implies from [10, Theorem 2.5] that $T$ possesses property $(g b)$. As $\Pi(T)=\Pi^{0}(T)=\emptyset$, then $T$ possesses property $(S B b)$. On the other hand, $\sigma_{S F_{+}^{-}}\left(T^{*}\right)=C(0,1)$. Thus $\Delta_{a}\left(T^{*}\right) \neq \Pi^{0}\left(T^{*}\right)$ and $T^{*}$ does not possess property $(b)$. Consequently $T^{*}$ does not possess property $(S B b)$.

Recall that an operator $T \in L(X)$ is said to possess property $(B w)$ if $\Delta^{g}(T)=E^{0}(T)$ and is said to possess property $(B b)$ if $\Delta^{g}(T)=\Pi^{0}(T)$, where $\Delta^{g}(T)=\sigma(T) \backslash \sigma_{B W}(T)$. The properties $(B w)$ and $(B b)$ have been introduced very recently in [15] and [19], respectively as variants of Weyl's and Browder's theorem. In the next theorem, we investigate the relationship between property $(S B w)$ and property $(B w)$.

Theorem 2.7. Let $T \in L(X)$. Then $T$ possesses property $(S B w)$ if and only if:

(i) $T$ possesses property $(B w)$;

(ii) $\operatorname{ind}(T-\lambda I)=0$ for all $\lambda \in \Delta_{a}^{g}(T)$; where $\operatorname{ind}(T-\lambda I)$ is the index of $T-\lambda I$. 
Proof. Suppose that $T$ possesses property $(S B w)$ and let $\lambda \in \Delta^{g}(T)$. Since $\sigma_{S B F_{+}^{-}}(T) \subset \sigma_{B W}(T)$, then $\lambda \notin \sigma_{S B F_{+}^{-}}(T)$. We also have that $\lambda \in \sigma_{a}(T)$. Indeed, if $\lambda \notin \sigma_{a}(T)$, as $\lambda \notin \sigma_{B W}(T)$, then $T-\lambda I$ will be invertible and this is impossible since $\lambda \in \sigma(T)$. As $T$ possesses property $(S B w)$, then $\lambda \in E^{0}(T)$. This implies that $\Delta^{g}(T) \subset E^{0}(T)$. To show the opposite inclusion, let $\lambda \in$ $E^{0}(T)$ be arbitrary. Since $T$ possesses property $(S B w)$, then $\lambda \notin \sigma_{S B F_{+}^{-}}(T)$ and so $\operatorname{ind}(T-\lambda I) \leq 0$. As $\lambda \in \operatorname{iso} \sigma(T)$, then $T^{*}$ has the SVEP at $\lambda$. By [7, Corollary 2.8], we deduce that ind $(T-\lambda I) \geq 0$. So ind $(T-\lambda I)=0$ and $\lambda \notin \sigma_{B W}(T)$. Hence $\Delta^{g}(T)=E^{0}(T)$ and ind $(T-\lambda I)=0$ for all $\lambda \in \Delta_{a}^{g}(T)$. Conversely, assume that $T$ possesses property $(B w)$ and ind $(T-\lambda I)=0$ for all $\lambda \in \Delta_{a}^{g}(T)$. If $\lambda \in \Delta_{a}^{g}(T)$, then $T-\lambda I$ is a semi-B-Fredholm operator such that $\operatorname{ind}(T-\lambda I)=0$. Thus $T-\lambda I$ is a B-Weyl operator. Since $T$ possesses property $(B w)$, then $\lambda \in E^{0}(T)$ and hence $\Delta_{a}^{g}(T) \subset E^{0}(T)$. Now if $\lambda \in E^{0}(T)$, then $T-\lambda I$ is a B-Weyl operator and $\lambda \in \sigma(T)$. Hence $\lambda \in \Delta_{a}^{g}(T)$ and $T$ possesses property $(S B w)$.

Similarly to Theorem 2.7, we have the following relationship between property $(S B b)$ and property $(B b)$ which we give without proof.

Theorem 2.8. Let $T \in L(X)$. Then $T$ possesses property $(S B b)$ if and only if:

(i) $T$ possesses property $(B b)$;

(ii) $\operatorname{ind}(T-\lambda I)=0$ for all $\lambda \in \Delta_{a}^{g}(T)$.

From Theorem 2.7 and Theorem 2.8, we have: the properties $(S B w)$ and $(S B b)$ imply the properties $(B w)$ and $(B b)$, respectively. But the following example shows that the converses do not hold in general.

Example 2.9. On the Banach space $\ell^{2}(\mathbb{N}) \oplus \ell^{2}(\mathbb{N})$ we consider the operator $T=0 \oplus R$ where $R$ is the unilateral right shift operator. Then $\sigma(T)=$ $\sigma_{B W}(T)=D(0,1)$ and $E^{0}(T)=\Pi^{0}(T)=\emptyset$. This implies that $\Delta^{g}(T)=$ $E^{0}(T)$ and $\Delta^{g}(T)=\Pi^{0}(T)$, i.e. $T$ possesses property $(B w)$ and property $(B b)$. On the other hand, $\sigma_{a}(T)=C(0,1) \cup\{0\}$ and $\sigma_{S B F_{+}^{-}}(T)=C(0,1)$. Thus $\Delta_{a}^{g}(T) \neq \Pi^{0}(T)$ and $\Delta_{a}^{g}(T) \neq E^{0}(T)$, i.e. $T$ does not possess either property $(S B w)$ nor property $(S B b)$. Here $\Delta_{a}^{g}(T)=\{0\}$ but ind $(T) \neq 0$.

Definition 2.10. A bounded linear operator $T \in L(X)$ is said to possess property $(S B a w)$ if $\Delta_{a}^{g}(T)=E_{a}^{0}(T)$ and is said to possess property $(S B a b)$ if $\Delta_{a}^{g}(T)=\Pi_{a}^{0}(T)$.

From Theorem 2.5 we deduce that property $(S B b)$ implies property $(S B a b)$, but the converse is not true in general as shown by the following example: let $T=R \oplus U$ be defined on the Banach space $\ell^{2}(\mathbb{N}) \oplus \ell^{2}(\mathbb{N})$ where $R$ is the unilateral right shift operator defined on $\ell^{2}(\mathbb{N})$, and $U$ is defined as 
above. Then $\sigma_{a}(T)=C(0,1) \cup\{0\}, \sigma_{S B F_{+}^{-}}(T)=C(0,1)$ and $\Pi_{a}^{0}(T)=\{0\}$. So $T$ possesses property $(S B a b)$. But it does not possess property $(S B b)$, since $\Pi^{0}(T)=\emptyset$. Observe that the two properties $(S B w)$ and $(S B a w)$ for $T \in L(X)$ are independent. Indeed, the example $R \oplus U$ defined above shows that property $(S B a w)$ does not imply property $(S B w)$ because $E_{a}^{0}(R \oplus U)=$ $\{0\}, E^{0}(R \oplus U)=\emptyset$. But the example $R \oplus S$ defined below (see Remark 2.16 ) shows that property $(S B w)$ does not imply property $(S B a w)$ because $E^{0}(R \oplus S)=\emptyset$.

However, we give in the next corollary conditions for the equivalence of two properties $(S B b)$ and $(S B a b)$.

Corollary 2.11. Let $T \in L(X)$. Then the following statements are equivalent.

(i) $T$ possesses property $(S B b)$;

(ii) $T$ possesses property $(S B a b)$ and $\Pi^{0}(T)=\Pi_{a}^{0}(T)$;

(iii) $T$ possesses property $(S B a b)$ and $\Pi^{0}(T)=\Pi_{a}(T)$.

Proof.

(i) $\Longleftrightarrow$ (ii) Suppose that $T$ possesses property $(S B b)$. By Theorem 2.5, $T$ possesses property $(b)$. This implies from $[11$, Corollary 2.7$]$ that $\Pi^{0}(T)=$ $\Pi_{a}^{0}(T)$. Thus $\Delta_{a}^{g}(T)=\Pi_{a}^{0}(T)$ and $T$ possesses property $(S B a b)$. The reverse implication is trivial.

(ii) $\Longleftrightarrow$ (iii) Follows directly by Theorem 2.5.

Theorem 2.12. Let $T \in L(X)$. Then $T$ possesses property (SBaw) if and only if $T$ satisfies a-Weyl's theorem and $\sigma_{S B F_{+}^{-}}(T)=\sigma_{S F_{+}^{-}}(T)$.

Proof. Suppose that $T$ possesses property ( $S B a w)$, that is $\Delta_{a}^{g}(T)=E_{a}^{0}(T)$. Since $\Delta_{a}(T) \subset \Delta_{a}^{g}(T)$ then $\Delta_{a}(T) \subset E_{a}^{0}(T)$. Now if $\lambda \in E_{a}^{0}(T)$, then $T-\lambda I$ is an upper semi-B-Fredholm operator. Since $\lambda$ is an eigenvalues of $T$ of finite multiplicity, by [11, Lemma 2.2] we have $T-\lambda I$ is an upper semiFredholm operator. Thus $\Delta_{a}(T) \supset E_{a}^{0}(T)$ and $\Delta_{a}(T)=E_{a}^{0}(T)$. So $T$ satisfies a-Weyl's theorem. We then have $\sigma_{S B F_{+}^{-}}(T)=\sigma_{a}(T) \backslash E_{a}^{0}(T)$ and $\sigma_{S F_{+}^{-}}(T)=\sigma_{a}(T) \backslash E_{a}^{0}(T)$. So $\sigma_{S B F_{+}^{-}}(T)=\sigma_{S F_{+}^{-}}(T)$.

Conversely, the condition $\sigma_{S B F_{+}^{-}}(T)=\sigma_{S F_{+}^{-}}(T)$ entails that $\Delta_{a}^{g}(T)=$ $\Delta_{a}(T)$ and a-Weyl's theorem for $T$ implies that $\Delta_{a}^{g}(T)=E_{a}^{0}(T)$. So $T$ possesses property (SBaw).

Remark 2.13. 1) The operator $T$ defined as in part 1) of Remark 2.6 shows that generally, a-Weyl's theorem does not imply the property $(S B a w)$. Indeed, since $\sigma_{S B F_{+}^{-}}(T)=E_{a}^{0}(T)=\emptyset, \sigma_{a}(T)=\sigma_{S F_{+}^{-}}(T)=\{0\}$ then $\Delta_{a}(T)=E_{a}^{0}(T)$, but $\Delta_{a}^{g}(T) \neq E_{a}^{0}(T)$. 
2) The property (SBaw) is not intermediate between a-Weyl's theorem and generalized a-Weyl's theorem. Indeed, we define $T$ on the Banach space $\ell^{2}(\mathbb{N}) \oplus \ell^{2}(\mathbb{N})$ by $T=I \oplus S$ where $I$ is the identity operator on $\ell^{2}(\mathbb{N})$ and $S$ is an injective quasinilpotent operator on $\ell^{2}(\mathbb{N})$ which is not nilpotent. Then $\sigma_{a}(T)=\{0,1\}, \sigma_{S B F_{+}^{-}}(T)=\{0\}, E_{a}(T)=\{1\}$ and $E_{a}^{0}(T)=\emptyset$. So $\Delta_{a}^{g}(T)=E_{a}(T)$, i.e. $T$ satisfies the generalized aWeyl's theorem, but it does not possess property $(S B a w)$ because $\Delta_{a}^{g}(T) \neq$ $E_{a}^{0}(T)$. On the other hand, let $Q$ be defined for each $x=\left(\xi_{i}\right) \in \ell^{1}$ by $Q\left(\xi_{1}, \xi_{2}, \xi_{3}, \ldots, \xi_{k}, \ldots\right)=\left(0, \alpha_{1} \xi_{1}, \alpha_{2} \xi_{2}, \ldots, \alpha_{k-1} \xi_{k-1}, \ldots\right)$, where $\left(\alpha_{i}\right)$ is a sequence of complex numbers such that $0<\left|\alpha_{i}\right| \leq 1$ and $\sum_{i=1}^{\infty}\left|\alpha_{i}\right|<\infty$ and define $T$ on $X=\ell^{1} \oplus \ell^{1}$ by $T=Q \oplus 0$. Then $\sigma_{a}(T)=\{0\}, E_{a}^{0}(T)=\emptyset$, $E_{a}(T)=\{0\}$ and $\sigma_{S B F_{+}^{-}}(T)=\{0\}$. Thus $\Delta_{a}^{g}(T)=E_{a}^{0}(T)$, i.e. $T$ possesses property $(S B a w)$, but it does not satisfy the generalized a-Weyl's theorem because $\Delta_{a}^{g}(T) \neq E_{a}(T)$.

3) The operator defined as in part 2) of Remark 2.3 shows that in general, property $(S B a w)$ is not transmitted from an operator to its dual.

Theorem 2.14. Let $T \in L(X)$. Then the following statements are equivalent:

(i) $T$ possesses property $(S B a b)$;

(ii) $T$ satisfies a-Browder's theorem and $\sigma_{S B F_{+}^{-}}(T)=\sigma_{S F_{+}^{-}}(T)$;

(iii) $T$ satisfies a-Browder's theorem and $\Pi_{a}^{0}(T)=\Pi_{a}(T)$.

Proof.

(i) $\Longleftrightarrow$ (ii) If $T$ possesses property $(S B a b)$, that is $\Delta_{a}^{g}(T)=\Pi_{a}^{0}(T)$, then $\Delta_{a}(T) \subset \Pi_{a}^{0}(T)$. As $\Delta_{a}(T) \supset \Pi_{a}^{0}(T)$ is always true, then $\Delta_{a}(T)=\Pi_{a}^{0}(T)$, i.e. $T$ satisfies a-Browder's theorem. We then have $\sigma_{S B F_{+}^{-}}(T)=\sigma_{a}(T) \backslash$ $\Pi_{a}^{0}(T)$ and $\sigma_{S F_{+}^{-}}(T)=\sigma_{a}(T) \backslash \Pi_{a}^{0}(T)$. So $\sigma_{S B F_{+}^{-}}(T)=\sigma_{S F_{+}^{-}}(T)$. The reverse implication is trivial.

(i) $\Longleftrightarrow$ (iii) If $T$ possesses property $(S B a b)$, then $T$ satisfies a-Browder's theorem. Hence $\Delta_{a}^{g}(T)=\Pi_{a}(T)=\Pi_{a}^{0}(T)$. Conversely, if $T$ satisfies aBrowder's theorem, then $T$ satisfies generalized a-Browder's theorem $\Delta_{a}^{g}(T)$ $=\Pi_{a}(T)$. As $\Pi_{a}(T)=\Pi_{a}^{0}(T)$ then $\Delta_{a}^{g}(T)=\Pi_{a}^{0}(T)$ and $T$ possesses property $(S B a b)$.

Corollary 2.15. Let $T \in L(X)$. Then the following statements are equivalent:

(i) $T$ possesses property (SBaw);

(ii) $T$ possesses property $(S B a b)$ and $E_{a}^{0}(T)=\Pi_{a}^{0}(T)$;

(iii) $T$ possesses property $(S B a b)$ and $E_{a}^{0}(T)=\Pi_{a}(T)$. 
Proof.

(i) $\Longrightarrow$ (ii) If $T$ satisfies property ( $S B a w)$, then from Theorem 2.12 and [8, Corollary 3.5] we conclude that $T$ satisfies a-Browder's theorem and $\sigma_{S B F_{+}^{-}}(T)=\sigma_{S F_{+}^{-}}(T)$. So $T$ possesses property $(S B a b)$ and $E_{a}^{0}(T)=\Pi_{a}^{0}(T)$.

(ii) $\Longrightarrow$ (iii) Obvious, see Theorem 2.14.

(iii) $\Longrightarrow$ (i) The property $(S B a b)$ entails that $\Delta_{a}^{g}(T)=\Pi_{a}(T)$. As $E_{a}^{0}(T)$ $=\Pi_{a}(T)$, then $\Delta_{a}^{g}(T)=E_{a}^{0}(T)$, a desired.

Remark 2.16. 1) From Theorem 2.14, we have property $(S B a b)$ for $T \in$ $L(X)$ implies a-Browder's theorem for $T$. But the operator $T=0$ shows that the converse is not true in general, since $\sigma_{a}(T)=\sigma_{S F_{+}^{-}}(T)=\{0\}$, $\Pi_{a}^{0}(T)=\emptyset$ and $\sigma_{S B F_{+}^{-}}(T)=\emptyset$. We also observe that $\Pi_{a}(T)=\{0\}$.

2) Similarly, we have by Corollary 2.15 that if $T \in L(X)$ possesses property $(S B a w)$ then it possesses property $(S B a b)$, but generally, the converse is not true as the following example shows: let $R \oplus S$ be defined on the Banach space $\ell^{2}(\mathbb{N}) \oplus \ell^{2}(\mathbb{N})$ where $R$ is the unilateral right shift operator and $S$ be defined on $\ell^{2}(\mathbb{N})$ by $S\left(x_{1}, x_{2}, x_{3}, \ldots\right)=\left(\frac{1}{2} x_{2}, \frac{1}{3} x_{3}, \ldots\right)$, then $\sigma_{a}(R \oplus S)=\sigma_{S B F_{+}^{-}}(R \oplus S)=C(0,1) \cup\{0\}, \Pi_{a}^{0}(R \oplus S)=\emptyset$ and $E_{a}^{0}(R \oplus S)=\{0\}$. This proves that $R \oplus S$ possesses property $(S B a b)$, but it does not possess property $(S B a w)$. Here $\Pi_{a}(R \oplus S)=\emptyset$.

\section{Summary OF RESUlts}

In this last part, we give a summary of known Weyl type theorems as in [8], including the properties introduced in $[1,11,12,17,19]$ and in this paper. We use the abbreviations $W, g W,(B w),(w),(g w),(S B w),(a w)$, (gaw), (Baw), aW, gaW and (SBaw) to signify that an operator $T \in L(X)$ obeys Weyl's theorem, generalized Weyl's theorem, property $(B w)$, property $(w)$, property $(g w)$, property $(S B w)$, property $(a w)$, property (gaw), property $(B a w)$, a-Weyl's theorem, generalized a-Weyl's theorem and property $(S B a w)$, respectively. Similarly, the abbreviations $B, g B, a B, g a B,(B b)$, $(B a b),(b),(g b),(a b),(g a b),(S B b)$ and $(S B a b)$ have analogous meaning with respect to Browder's theorem or the properties introduced in [11] or the properties introduced in [12] or the properties introduced in [19] or the new properties introduced in this paper.

In the following diagram, which extends the similar diagram given in [12], arrows signify implications between Weyl type theorems, Browder type theorems, property $(B w)$, property $(B b)$, property $(B a w)$, property $(B a b)$, property $(S B w)$, property $(S B b)$, property $(S B a w)$ and property $(S B a b)$. The numbers near the arrows are references to the results in the present paper (numbers without brackets) or to the bibliography therein (the numbers in square brackets). 


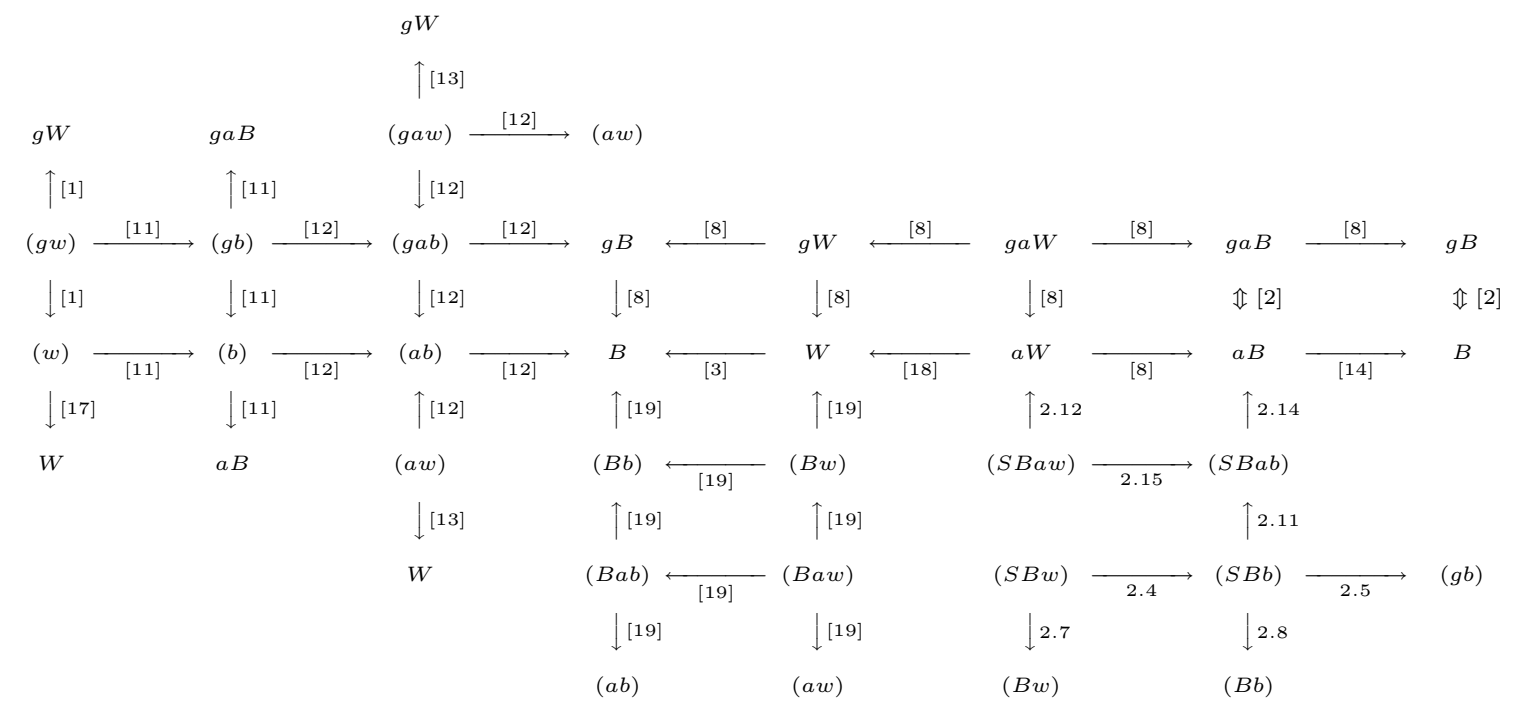

Table 1 
The following table summarizes the meaning of various theorems and properties.

\begin{tabular}{|l|l||l|l|}
\hline$W$ & $\sigma(T) \backslash \sigma_{W}(T)=E^{0}(T)$ & $B$ & $\sigma(T) \backslash \sigma_{W}(T)=\Pi^{0}(T)$ \\
$g W$ & $\sigma(T) \backslash \sigma_{B W}(T)=E(T)$ & $g B$ & $\sigma(T) \backslash \sigma_{B W}(T)=\Pi(T)$ \\
$(B w)$ & $\sigma(T) \backslash \sigma_{B W}(T)=E^{0}(T)$ & $(B b)$ & $\sigma(T) \backslash \sigma_{B W}(T)=\Pi^{0}(T)$ \\
$(w)$ & $\sigma_{a}(T) \backslash \sigma_{S F_{+}^{-}}(T)=E^{0}(T)$ & $(b)$ & $\sigma_{a}(T) \backslash \sigma_{S F_{+}^{-}}(T)=\Pi^{0}(T)$ \\
$(g w)$ & $\sigma_{a}(T) \backslash \sigma_{S B F_{+}^{-}}(T)=E(T)$ & $(g b)$ & $\sigma_{a}(T) \backslash \sigma_{S B F_{+}^{-}}(T)=\Pi(T)$ \\
$(S B w)$ & $\sigma_{a}(T) \backslash \sigma_{S B F_{+}^{-}}(T)=E^{0}(T)$ & $(S B b)$ & $\sigma_{a}(T) \backslash \sigma_{S B F_{+}^{-}}(T)=\Pi^{0}(T)$ \\
$(a w)$ & $\sigma(T) \backslash \sigma_{W}(T)=E_{a}^{0}(T)$ & $(a b)$ & $\sigma(T) \backslash \sigma_{W}(T)=\Pi_{a}^{0}(T)$ \\
$(g a w)$ & $\sigma(T) \backslash \sigma_{B W}(T)=E_{a}(T)$ & $(g a b)$ & $\sigma(T) \backslash \sigma_{B W}(T)=\Pi_{a}(T)$ \\
$(B a w)$ & $\sigma(T) \backslash \sigma_{B W}(T)=E_{a}^{0}(T)$ & $(B a b)$ & $\sigma(T) \backslash \sigma_{B W}(T)=\Pi_{a}^{0}(T)$ \\
$a W$ & $\sigma_{a}(T) \backslash \sigma_{S F_{+}^{-}}(T)=E_{a}^{0}(T)$ & $a B$ & $\sigma_{a}(T) \backslash \sigma_{S F_{+}^{-}}(T)=\Pi_{a}^{0}(T)$ \\
$g a W$ & $\sigma_{a}(T) \backslash \sigma_{S B F_{+}^{-}}(T)=E_{a}(T)$ & $g a B$ & $\sigma_{a}(T) \backslash \sigma_{S B F_{+}^{-}}(T)=\Pi_{a}(T)$ \\
$(S B a w)$ & $\sigma_{a}(T) \backslash \sigma_{S B F_{+}^{-}}(T)=E_{a}^{0}(T)$ & $(S B a b)$ & $\sigma_{a}(T) \backslash \sigma_{S B F_{+}^{-}}(T)=\Pi_{a}^{0}(T)$ \\
\hline
\end{tabular}

Table 2

\section{REFERENCES}

[1] M. Amouch, M. Berkani, On the property (gw), Mediterr. J. Math., 3 (2008), 371-378.

[2] M. Amouch, H. Zguitti, On the equivalence of Browder's and generalized Browder's theorem, Glasgow Math. J., 48 (2006), 179-185.

[3] B. A. Barnes, Riesz points and Weyl's theorem, Integral Equations Oper. Theory, 34 (1999), 187-196.

[4] M. Berkani, On a class of quasi-Fredholm operators, Integral Equations Oper. Theory, 34 (2) (1999), 244-249.

[5] M. Berkani, Index of B-Fredholm operators and generalization of a Weyl theorem. Proc. Amer. Math. Soc., 130 (6) (2002), 1717-1723.

[6] M. Berkani, B-Weyl spectrum and poles of the resolvent, J. Math. Anal. Appl., 272 (2) (2002), 596-603.

[7] M. Berkani and N. Castro and S. V. Djordjević, Single valued extension property and generalized Weyl's theorem, Math. Bohemica, 131 (1) (2006), 29-38.

[8] M. Berkani, J.J. Koliha, Weyl type theorems for bounded linear operators, Acta Sci. Math., (Szeged), 69 (2003), 359-376.

[9] M. Berkani, M. Sarih, On semi B-Fredholm operators, Glasgow Math. J., 43 (2001), $457-465$

[10] M. Berkani, M. Sarih and H. Zariouh, Browder-type Theorems and SVEP, Mediterr. J. Math., 8 (2011), 399-409.

[11] M. Berkani, H. Zariouh, Extended Weyl type theorems, Math. Bohemica, 134 (4) (2009), 369-378. 
[12] M. Berkani, H. Zariouh, New extended Weyl type theorems, Mat. Vesnik, 62 (2) (2010), $145-154$.

[13] M. Berkani, H. Zariouh, Perturbation results for Weyl type theorems, Acta Math. Univ. Comenianae, 80 (2011), 119-132.

[14] S. V. Djordjević and Y. M. Han, Browder's theorems and spectral continuity, Glasgow Math. J., 42 (2000), 479-486.

[15] A. Gupta, N. Kashyap, Property (Bw) and Weyl type theorems, Bull. Math. Anal. Appl., 3 (2) (2011), 1-7.

[16] K. B. Laursen and M. M. Neumann, An Introduction to Local Spectral Theory, Clarendon Press Oxford, (2000)

[17] V. Rakočević, On a class of operators, Mat. Vesnik, 37 (1985), 423-426.

[18] V. Rakočević, Operators obeying a-Weyl's theorem, Rev. Roumaine Math. Pures Appl., 34 (1989), 915-919.

[19] H. Zariouh, H. Zguitti, Variations on Browder's theorem, Acta Math. Univ. Comenianae, 81 (2) (2012), 255-264.

(Received: May 2, 2012)

(Revised: August 15, 2012)
Mohammed Berkani, Mohammed Kachad Department of Mathematics

Science faculty of Oujda

University Mohammed I

Operator Theory Team

Morocco

berkanimo@aim.com

kachad.mohammed@gmail.com

Hassan Zariouh

Centre régional des métiers de l'éducation et de la formation, B.P 458 Oujda, Morocco et Equipe de la Théorie des Oprateurs

Université Mohammed Premier

Faculté des Sciences

Dépt. de Mathématiques et Informatique

Morocco

h.zariouh@yahoo.fr

Hassane Zguitti

Dépt. de Mathématiques et Informatique Faculté Pluridisciplinaire de Nador

Université Mohammed Premier

B.P 300 Selouane 62700, Nador

Morocco

zguitti@hotmail.com 